\title{
MULTIDIMENSIONAL LIMIT THEOREMS FOR HOMOGENEOUS SUMS: A SURVEY AND A GENERAL TRANSFER PRINCIPLE
}

\author{
Ivan Nourdin ${ }^{1}$, Giovanni Peccati $^{1}$, Guillaume Poly $^{2}$ and Rosaria Simone ${ }^{3}$
}

\begin{abstract}
We provide a synthetic yet comprehensive review of the so-called fourth moment criterion, and of universal limit theorems, for multilinear homogeneous sums, in both the classical and the free probability settings. In addition to such a general picture, we also prove a novel multidimensional transfer principle for Central Limit Theorems involving homogeneous sums with leptokurtic or mesokurtic entries. The key step will be to prove that joint and component-wise convergence are indeed equivalent for these random objects, encompassing well-known results concerning Wiener and Wigner Chaoses.
\end{abstract}

Mathematics Subject Classification. 60F17, 60F05, 46L54.

Received June 24, 2015. Revised February 15, 2016. Accepted May 19, 2016.

\section{INTRODUCTION}

The fourth moment phenomenon is a collection of probabilistic results, allowing one to deduce central and non central limit theorems for a sequence $\left\{X_{n}: n \geq 1\right\}$ of non-linear functionals of a random field, under the sole condition that the both sequences $\left\{\mathbb{E}\left[X_{n}^{2}\right]: n \geq 1\right\}$ and $\left\{\mathbb{E}\left[X_{n}^{4}\right]: n \geq 1\right\}$ converge towards the corresponding two first even moments of the target distribution. First discovered in [33] in the context of non-linear transformations of Gaussian fields, such a phenomenon has gained an increasing interest in the mathematical community, due to its wide range of applications. The reader is referred to the monograph [26] for a comprehensive introduction to the Malliavin-Stein method for normal approximations, as well as to $[12,13,34]$ for recent surveys, and to the online resource ${ }^{4}$ for a constantly updated repository of the mathematical literature on this topic.

Such a theory has also a counterpart in the framework of non-commutative probability theory. This line of research started with the paper [20], where the authors exploited the non-commutative version of the Malliavin calculus introduced [8] in order to derive a free version of the Fourth Moment Theorem for elements of the Wigner Chaos. See $[2,9,10,15-17,30,32,40]$, as well as the discussion below, for recent developments.

\footnotetext{
Keywords and phrases. Fourth moment phenomenon, free probability, homogeneous sums, multidimensional limit theorems, wiener chaos, wigner chaos.

1 Université du Luxembourg, Faculté des Sciences, de la Technologie et de la Communication, UR en Mathématiques, 6, rue Richard Coudenhove-Kalergi, 1359 Luxembourg. ivan.nourdin@uni.lu; giovanni.peccati@uni.lu

2 Unité de Formation et de Recherche Mathématiques MATH, Institut de Recherche Mathématiques de Rennes (IRMAR), 263 Avenue du General Leclerc, Building: B022, CS 74205, 35042 Rennes cedex. guillaume.poly@univ-rennes1.fr

3 Dipartimento di Scienze Politiche, Università degli Studi di Napoli - Federico II, Via L. Rodinò 22, 80138 Napoli, Italy. rosaria.simone@unina.it

${ }^{4}$ https://sites.google.com/site/malliavinstein/home.
} 
Another remarkable phenomenon concerning both the Wiener and Wigner homogeneous chaoses is their universal asymptotic behavior as to central convergence - see $[16,28,38,40]$ - that is tightly connected to the Lindeberg-type method of influence functions, established in [23, 28] (in the classical setting), and in [16] (in the non-commutative setting). Universal limit theorems have relevance in a wide range of research areas: for instance, limit theorems encompassing the universality phenomenon have been investigated recently in dealing with the random graphs colouring problem [7].

Our focus in this paper is on multilinear homogeneous sums in independent random variables, in both the classical and the non-commutative frameworks. Three aspects for these random objects will be considered and presented in a unified manner: the fourth moment phenomenon, the universal behaviour as to central and noncentral convergence, and the equivalence between joint and component-wise convergence in the multidimensional CLT. All these properties are known to hold for elements in the Wiener and the Wigner chaoses - see e.g. [30]. In what follows, we will analyze these topics in the light of the recent reference [32], where an extended version of the Fourth Moment Theorems and of the universality principle for homogeneous sums in independent random variables with non-negative fourth cumulant was proved, thus encompassing the Gaussian and semicircular cases.

The last section of this work will contain some important new results, showing how the one-dimensional Theorems 3.5 and 3.6 can be nicely extended to the more challenging multidimensional setting. More precisely, the final outcome of our discussion are Theorem 4.1 and its free version Theorem 4.2, in which we shall prove that joint and component-wise central convergence are equivalent for vectors of homogeneous sums in independent copies of leptokurtic or mesokurtic variables, both in the commutative and in the non-commutative frameworks. The combination of these results will lead eventually to Theorem 4.3, that is a general transfer principle for (vectors of) such random variables, extending the transfer principle for the CLT between Wiener and Wigner chaoses provided in [30]. Results connecting the commutative and the free probability theories can be potentially very useful for establishing free counterparts to well-understood results in the classical setting: for instance, a remarkable example is given by the free version of the Breuer-Major Theorem pointed out in [20].

We would like to stress that the proofs we provide do not require any additional techniques with respect to those developed in [32], of which this note is meant to be a natural sequel.

\section{NOTATIONS AND PRELIMINARY DEFINITIONS}

Let us start by setting some preliminary notations and definitions, basically borrowed from the benchmark reference [32], which will constitute the basis of the whole discussion. For any unexplained concept or result pertaining to free probability theory, the reader is referred to the fundamental references $[24,41]$.

For every $n \in \mathbb{N}$, set $[n]:=\{1, \ldots, n\}$. Let $X$ be a random variable defined on a fixed probability space $(\Omega, \mathcal{F}, \mathbb{P})$. Unless otherwise specified, it will be always assumed that $X$ satisfies the following assumptions, that will be referred to as Assumption (1):

(i) $X$ is centered and has unit variance;

(ii) $\mathbb{E}\left[X^{3}\right]=0$;

(iii) there exists $\epsilon>0$ such that $\mathbb{E}\left[|X|^{4+\epsilon}\right]<\infty$.

Given a sequence $\mathbf{X}=\left\{X_{i}\right\}_{i \geq 1}$ of independent copies of $X$ (i.i.d. for short), we will consider random variables having the form of multilinear homogeneous polynomials ${ }^{5}$ of degree $d \geq 2$ :

$$
Q_{\mathbf{X}}(f)=\sum_{i_{1}, \ldots, i_{d}=1}^{n} f\left(i_{1}, \ldots, i_{d}\right) X_{i_{1}} \cdots X_{i_{d}},
$$

where the mapping $f:[n]^{d} \rightarrow \mathbb{R}$ is an admissible kernel, in the sense of the following definition.

\footnotetext{
${ }^{5}$ For short, homogeneous sums.
} 
Definition 2.1. For a given degree $d \geq 2$ and some integer $n \geq 1$, a function $f:[n]^{d} \rightarrow \mathbb{R}$ is said to be an admissible kernel if the following properties are satisfied:

(i) $f$ vanishes on diagonals, that is, $f\left(i_{1}, \ldots, i_{d}\right)=0$ whenever $i_{j}=i_{k}$ for some $k \neq j$;

(ii) $f$ is symmetric, namely $f\left(i_{1}, \ldots, i_{d}\right)=f\left(i_{\sigma(1)}, \ldots, i_{\sigma(d)}\right)$ for any permutation $\sigma$ of $[d]$ and any $\left(i_{1}, \ldots, i_{d}\right) \in$ $[n]^{d}$;

(iii) $f$ satisfies the normalization:

$$
d ! \sum_{i_{1}, \ldots, i_{d}=1}^{n} f\left(i_{1}, \ldots, i_{d}\right)^{2}=1 .
$$

Whenever $f$ is an admissible kernel and $X$ satisfies Assumption (1), the homogeneous sum $Q_{\mathbf{X}}(f)$ verifies $\mathbb{E}\left[Q_{\mathbf{X}}(f)\right]=0$ and $\mathbb{E}\left[Q_{\mathbf{X}}(f)^{2}\right]=1$. We stress that the symmetry and the normalization assumptions on $f$ are introduced for mere convenience: indeed, given a function $f:[n]^{d} \rightarrow \mathbb{R}$ that is vanishing on diagonals, it is always possible to generate an admissible kernel $\tilde{f}$ by first symmetrizing $f$ and then by properly renormalizing it.

Starting from de Jong's criterion [14], a relevant role in the task of assessing the central convergence of homogeneous sums as in (1) has been played by influence functions, measuring the impact of each variable $X_{i}$ on the overall fluctuations of $Q_{\boldsymbol{X}}\left(f_{n}\right)[23,26]$.

Definition 2.2. Given an admissible kernel $f_{n}:[n]^{d} \rightarrow \mathbb{R}$, the $i$ th influence function of $f_{n}$ is defined by:

$$
\operatorname{Inf}_{i}\left(f_{n}\right):=\sum_{i_{2}, \ldots, i_{d}=1}^{n} f_{n}\left(i, i_{2}, \ldots, i_{d}\right)^{2} .
$$

As already highlighted in the Introduction, the ultimate goal of the present note is to supplement the findings of [32], by providing an extension of the results therein established to the multidimensional setting. In order to meet such intent, we shall need the following definitions. $N \sim \mathcal{N}(0,1)$ will denote that $N$ is a random variable with the standard Gaussian distribution.

Definition 2.3. Let $\mathbf{X}=\left\{X_{i}\right\}_{i \geq 1}$ be a sequence of independent copies of a random variable $X$ verifying Assumption (1).

(a) We say that $X$ satisfies the Fourth Moment Theorem at the order $d \geq 2$ (for normal approximations of homogeneous sums) if, for every sequence $f_{n}:[n]^{d} \rightarrow \mathbb{R}$ of admissible kernels, the following statements are equivalent for $n \rightarrow \infty$ :

(i) $Q_{\mathbf{X}}\left(f_{n}\right) \stackrel{\text { Law }}{\longrightarrow} \mathcal{N}(0,1)$.

(ii) $\mathbb{E}\left[Q_{\mathbf{X}}\left(f_{n}\right)^{4}\right] \longrightarrow \mathbb{E}\left[N^{4}\right]=3$, where $N \sim \mathcal{N}(0,1)$.

(b) $X$ is said to be universal at the order $d$ (for normal approximations of homogeneous sums) if, for any sequence $f_{n}:[n]^{d} \rightarrow \mathbb{R}$ of admissible kernels, $Q_{\mathbf{X}}\left(f_{n}\right) \stackrel{\text { Law }}{\longrightarrow} \mathscr{N}(0,1)$ implies, as $n \rightarrow \infty$, the following Lindeberg-type condition:

$$
\tau_{n}:=\max _{i=1, \ldots, n} \operatorname{Inf}_{i}\left(f_{n}\right) \rightarrow 0 .
$$

Remark 2.4. We stress that, due to the normalization assumption on the admissible kernel $f_{n}, \tau_{n}\left(f_{n}\right) \leq 1$ : this justifies the fact that often one speaks about invariance principle for low-influence functions.

We now turn to the non-commutative setting. Consider a fixed $W^{*}$-probability space $(\mathcal{A}, \varphi)$, where $\mathcal{A}$ is a von Neumann algebra of operators, and $\varphi$ is a unital, faithful and positive trace. Let $Y$ be a random variable on it, centered and with unit variance, that is, $\varphi(Y)=0$ and $\varphi\left(Y^{2}\right)=1$. In this case, it will be said for short that $Y$ satisfies assumption (2). 
If $\mathbf{Y}=\left\{Y_{i}\right\}_{i \geq 1}$ is a sequence of freely independent copies of $Y$, the free counterpart to random variables of the form (1) are self-adjoint elements of the type:

$$
Q_{\mathbf{Y}}(f)=\sum_{i_{1}, \ldots, i_{d}=1}^{n} f\left(i_{1}, \ldots, i_{d}\right) Y_{i_{1}} \cdots Y_{i_{d}}
$$

being $f$ an admissible kernel. From assumption (2) and the properties of $f$, it follows that $\varphi\left(Q_{\mathbf{Y}}(f)\right)=0$ and $d ! \varphi\left(Q_{\mathbf{Y}}(f)^{2}\right)=1$.

Remark 2.5. In the free setting, the natural choice for the coefficient of a homogeneous sum would be a mirror symmetric function, namely a kernel $f:[n]^{d} \rightarrow \mathbb{C}$ such that $f\left(i_{1}, i_{2}, \ldots, i_{d}\right)=\overline{f\left(i_{d}, \ldots, i_{2}, i_{1}\right)}$ for every $i_{1}, \ldots, i_{d} \in[n]$, with $\bar{z}$ denoting the complex conjugate of $z$. This assumption is the weakest possible to ensure that the element $Q_{\mathbf{Y}}(f)$ is self-adjoint. However, the forthcoming discussion will heavily rely on the universality property of the Wigner Semicircle law that has been so far established only for homogeneous sums with symmetric real-valued coefficients: indeed, both in ([9], Thm. 1.8) and in [16], counterexamples to the universality for mirror symmetric kernels have been provided.

For several reasons, the semicircular distribution is considered as the non-commutative analogue of the Gaussian distribution: for instance, it is the limit law for the free version of the Central Limit Theorem, so that semicircular approximation is customarily referred to as central approximation in the free sense.

As in the classical setting, we will be concerned by the following properties for a free random variable $Y$.

Definition 2.6. Let $Y$ satisfy assumption (2) and let $S$ be a standard semicircular random variable, for short $S \sim \mathcal{S}(0,1)$. Let $\boldsymbol{Y}=\left\{Y_{i}\right\}_{i \geq 1}$ and $\boldsymbol{S}=\left\{S_{i}\right\}_{i \geq 1}$ be sequences of freely independent copies of $Y$ and $S$ respectively. For a fixed $d \geq 2$ and for every $n \geq 1$, let $f_{n}:[n]^{d} \rightarrow \mathbb{R}$ be an admissible kernel.

(a) We say that $Y$ satisfies the free Fourth Moment Theorem at the order $d$ (for semicircular approximations of free homogeneous sums) if, for any sequence of admissible kernels $f_{n}:[n]^{d} \rightarrow \mathbb{R}$, the following statements are equivalent as $n \rightarrow \infty$ :

(i) $d !^{2} \varphi\left(Q_{\mathbf{Y}}\left(f_{n}\right)^{4}\right) \rightarrow \varphi\left(S^{4}\right)=2, \quad S \sim \mathcal{S}(0,1)$;

(ii) $\sqrt{d !} Q_{\mathbf{Y}}\left(f_{n}\right) \stackrel{\text { Law }}{\longrightarrow} \mathcal{S}(0,1)$.

(b) We say that $Y$ is free universal at the order $d$ (for semicircular approximations of free homogeneous sums) if, for any sequence $f_{n}:[n]^{d} \rightarrow \mathbb{R}$ of admissible kernels, $\sqrt{d !} Q_{\mathbf{Y}}\left(f_{n}\right) \stackrel{\text { Law }}{\longrightarrow} \mathcal{S}(0,1)$ implies, as $n \rightarrow \infty$,

$$
\tau_{n}=\max _{i=1, \ldots, n} \operatorname{Inf}_{i}\left(f_{n}\right) \rightarrow 0
$$

\section{State OF THE ART}

Our intent is dealing with the following three properties for multilinear homogeneous sums as in (1) and (2):

(a) the Fourth Moment Theorem, both as to central and non-central convergence;

(b) the universal asymptotic behavior for central convergence, for homogeneous sums with low-influence coefficients;

(c) equivalence between component-wise and joint convergence for the CLT in the multidimensional setting,

which now we will shortly comment. In order to provide a unified presentation, and due to the close connection between the Fourth Moment Theorem and the invariance principle based on the Lindeberg method of influence functions, we consider the first two topics together. The discussion will simultaneously consider commutative and free probability spaces, in order to enhance differences as well as analogies. 


\subsection{Fourth moment theorem and universality}

The focus here will be initially on Gaussian and Semicircular homogeneous sums. Let us start with the commutative framework.

A substantial simplification of the classical method of moments and cumulants for determining the central convergence in law of a sequence of homogeneous sums has been provided by the classical result known as de Jong's criterion [14], which is the first central limit theorem based on a fourth moment condition (and hence consisting in a partial fourth moment criterion).

Theorem 3.1. Let $d \geq 2$. If $\boldsymbol{X}=\left\{X_{i}\right\}_{i \geq 1}$ are independent, $\mathbb{E}\left[X_{i}\right]=0, \mathbb{E}\left[X_{i}^{2}\right]=1, \mathbb{E}\left[X_{i}^{4}\right]<\infty$, assume that, as $n \rightarrow \infty$ :

(1) $\mathbb{E}\left[Q_{\boldsymbol{X}}\left(f_{n}\right)^{4}\right] \longrightarrow \mathbb{E}\left[N^{4}\right]=3$, with $N \sim \mathcal{N}(0,1)$;

(2) $\tau_{n}:=\max _{i=1, \ldots, n} \operatorname{Inf}_{i}\left(f_{n}\right) \longrightarrow 0$.

Then $Q_{\boldsymbol{X}}\left(f_{n}\right) \stackrel{\text { Law }}{\longrightarrow} \mathcal{N}(0,1)$.

After Theorem 3.1, seeking for a pure fourth moment condition means dropping the condition on the low influence required to the coefficients.

For Wiener Chaos, de Jong's criterion has been considerably improved since the Nualart-Peccati criterion [33], where the authors, giving the birth to the so-called Malliavin-Stein Method, proved that this task is possible, and involves assessing only the convergence of the first two even moments. As a matter of fact, Point (a) in our Definition 2.3 has been inspired by the Fourth Moment Theorem for Gaussian Wiener Chaos. As shown originally in ([28], Thm. 1.2), if $X$ is normally distributed, then it verifies also point (b) in Definition 2.3. In this case, the corresponding homogeneous sums $Q_{\mathbf{X}}\left(f_{n}\right)$ are said to be elements of the $d$ th (Gaussian) Wiener chaos. A very comprehensive and detailed introduction to these concepts is the book [35], dealing also with the combinatorial foundations of the subject.

A crucial role in the proof of the universal behavior of the Gaussian Wiener chaos has been played by the findings in [23], giving rise to the Lindeberg method of influence functions. As a result, the authors proved that the proximity in law between homogeneous sums can be measured in terms of influence functions, as recorded in the next statement.

Theorem 3.2. Let $\boldsymbol{X}=\left\{X_{i}\right\}_{i \geq 1}$ and $\mathbf{Y}=\left\{Y_{i}\right\}_{i \geq 1}$ be sequences of independent centered random variables on a fixed probability space, with unit variance and uniformly bounded moments of every order. Then, for $d \geq 1$ and for every sequence of admissible kernels $f_{n}:[n]^{d} \rightarrow \mathbb{R}$,

$$
\mathbb{E}\left[Q_{\boldsymbol{X}}\left(f_{n}\right)^{m}\right]-\mathbb{E}\left[Q_{\boldsymbol{Y}}\left(f_{n}\right)^{m}\right]=\mathcal{O}\left(\sqrt{\tau_{n}}\right) \quad \forall m \in \mathbb{N},
$$

where $\tau_{n}:=\max _{i=1, \ldots, n} \operatorname{Inf}_{i}\left(f_{n}\right)$.

Remark 3.3. It is well known that the issue of universality is relevant only for homogeneous sums of degree $d \geq 2$, since no invariance principle holds for homogeneous sums of degree $d=1$ (see [28], for instance). Theorem 3.2 encompasses well-known results when $d=1$ : in this case, the $i$ th influence function is simply set to $\operatorname{Inf}_{i}\left(f_{n}\right)=f_{n}(i)^{2}$, so that $\sqrt{\tau_{n}}=\max _{i=1, \ldots, n}\left|f_{n}(i)\right|$ (see for instance [26], Prop. 11.1.3).

For degrees $d \geq 2$ and in view of Theorem 3.2, one could alternatively define $X$ to be universal at the order $d$ if, for any sequence $f_{n}:[n]^{d} \rightarrow \mathbb{R}$ of admissible kernels, $Q_{\mathbf{X}}\left(f_{n}\right) \stackrel{\text { Law }}{\longrightarrow} \mathscr{N}(0,1)$ implies $Q_{\mathbf{Z}}\left(f_{n}\right) \stackrel{\text { Law }}{\longrightarrow} \mathscr{N}(0,1)$ for every sequence $\mathbf{Z}$ of i.i.d. centered random variables, with unit variance.

For the non-commutative framework, the findings in ([20], Thm. 1.3) paved the way to the investigation of the fourth moment phenomenon by proving that Wigner integrals satisfy Definition 2.6 - Point (a). Later, in ([16], Thm. 1.4), the authors proved that simple Wigner integrals having the form of semicircular homogeneous 
sums satisfy Definition 2.6 - Point (b), as well. The starting point has been the forthcoming Theorem 3.4, recalling ([16], Thm. 1.3), where the authors extended Theorem 3.2 in the free probability setting, for multilinear homogeneous polynomials in freely independent variables.

Theorem 3.4. Let $(\mathcal{A}, \varphi)$ be a $W^{*}$-probability space. Let $\mathbf{X}=\left\{X_{i}\right\}_{i \geq 1}$ and $\mathbf{Y}=\left\{Y_{i}\right\}_{i \geq 1}$ be two sequences of centered freely independent random variables, with unit variance, such that $\mathbf{X}$ and $\mathbf{Y}$ are freely independent. Assume, moreover, that the elements of $\mathbf{X}$ (respectively $\mathbf{Y}$ ) have uniformly bounded moments, that is, for every $r \geq 1$ :

$$
\sup _{i \geq 1} \varphi\left(\left|X_{i}\right|^{r}\right)<\infty \quad\left(\operatorname{resp} . \sup _{i \geq 1} \varphi\left(\left|Y_{i}\right|^{r}\right)<\infty\right) .
$$

Set $d \geq 1$, and let $f_{n}:[n]^{d} \rightarrow \mathbb{R}$ be an admissible coefficient as in Definition 2.1. Then, for any integer $m \geq 1$ :

$$
\varphi\left(Q_{\boldsymbol{X}}\left(f_{n}\right)^{m}\right)-\varphi\left(Q_{\boldsymbol{Y}}\left(f_{n}\right)^{m}\right)=\mathcal{O}\left(\sqrt{\tau_{n}}\right),
$$

where $\tau_{n}:=\tau\left(f_{n}\right)=\max _{i=1, \ldots, n} \operatorname{Inf}_{i}\left(f_{n}\right)$.

By virtue of Theorem 3.4, the definition of free universal law can be equivalently formulated by saying that $Y$ is freely universal (at the order $d$ ) if, for any sequence $f_{n}:[n]^{d} \rightarrow \mathbb{R}$ of admissible kernels, $\sqrt{d !} Q_{\mathbf{Y}}\left(f_{n}\right) \stackrel{\text { Law }}{\longrightarrow} \mathcal{S}(0,1)$ implies $\sqrt{d !} Q_{\mathbf{Z}}\left(f_{n}\right) \stackrel{\text { Law }}{\longrightarrow} \mathcal{S}(0,1)$ for every sequence $\mathbf{Z}=\left\{Z_{i}\right\}_{i \geq 1}$ of freely independent and identically distributed random variables verifying assumption (2).

A first attempt to answer to the question: "What is so special about the Gaussian and the Semicircular distribution that entails both the fourth moment and the universality phenomena?", was run by the authors in [32]. The main results are summarized in Theorems 3.5 and 3.6 below, that provide the initial impetus for our purposes. To fix the notation, $\chi_{4}(X)=\mathbb{E}\left[X^{4}\right]-3$ and $\kappa_{4}(Y)=\varphi\left(Y^{4}\right)-2$ will denote, respectively, the fourth cumulant of a random variable $X$ satisfying assumption (1) and the fourth free cumulant of a non-commutative random variable $Y$ verifying assumption (2).

Theorem 3.5. Fix $d \geq 2$ and let $X$ be a random variable satisfying assumption (1). If $\mathbb{E}\left[X^{4}\right] \geq 3$ (or, equivalently, $\chi_{4}(X) \geq 0$ ), then $X$ satisfies the Fourth Moment Theorem and its law is universal at the order $d$ for normal approximations of homogeneous sums, in the sense of Definition 2.3.

Theorem 3.6. Fix $d \geq 2$ and consider a random variable $Y$ verifying assumption (2) and such that $\varphi\left(Y^{4}\right) \geq 2$ (or, equivalently, $\kappa_{4}(Y) \geq 0$ ). Then, $Y$ satisfies the free Fourth Moment Theorem and it is free universal at the order d for semicircular approximations of free homogeneous sums.

Remark 3.7. The conditions required by Theorems 3.5 and 3.6 are natural in the light of the notion of Kurtosis which is central in probability theory and statistics. We recall that the kurtosis of a random variable $X$ is the quantity $\chi_{4}(X) / \chi_{2}(X)^{2}$ which measures the fatness of the tail of the law of $X$. Leptokurtic random variables are such that $\chi_{4}(X)>0$ and mesokurtic ones satisfy $\chi_{4}(X)=0$. By extension, in the non-commutative case, our assumptions implicitly involve an analogous notion of the free kurtosis.

For the sake of completeness, we stress that the proofs of Theorems 3.5 and 3.6 both rely on combinatorial formulae for the fourth moment of a homogeneous sum expressed with respect to its Gaussian or semicircular counterparts. In the same reference [32], the same approach has been adapted to formulate Theorem 3.5 and 3.6 for homogeneous sums in independent possibly not identically distributed random variables and also for noncentral convergence (Gamma and free Poisson, respectively) (these results will be shortly presented in the next subsection). Moreover, the discussion of the existence of an optimal threshold for the fourth moment of $X$ (resp. of $Y$ ) that enables both the fourth moment criterion and the universality property has also been addressed (see [32], Thms. 5.1 and 5.2). In this regard, though aware that the thresholds $\mathbb{E}\left[X^{4}\right] \geq 3$ and $\varphi\left(Y^{4}\right) \geq 2$ are not optimal, we content ourselves to limit to these conditions, leaving for future works further investigation on this topic. 


\section{Non-central convergence}

The dual side of our investigation concerns the determination of the admissible limit laws for sequences of homogeneous sums in both a fourth moment type statement and with respect to which one observes a universal behavior.

So far, the only non-central commutative fourth-moment theorem involves Gamma limits: for $\nu>0$, let $G\left(\frac{\nu}{2}\right)$ denote a random variable with the Gamma distribution $\Gamma\left(\frac{\nu}{2}, 1\right)$, and set $F(\nu) \stackrel{\text { Law }}{=} 2 G\left(\frac{\nu}{2}\right)-\nu$, so that

$$
\mathbb{E}[F(\nu)]=0, \mathbb{E}\left[F(\nu)^{2}\right]=2 \nu, \mathbb{E}\left[F(\nu)^{3}\right]=8 \nu, \mathbb{E}\left[F(\nu)^{4}\right]=12 \nu^{4}+48 \nu .
$$

Note that if $\nu$ is a positive integer, then $F(\nu)$ has the centered $\chi_{\nu}^{2}$ distribution with $\nu$ degrees of freedom, namely, $F(\nu) \stackrel{\text { Law }}{=} \sum_{i=1}^{\nu}\left(N_{i}-1\right)$, where $N_{1}, \ldots, N_{\nu}$ are i.i.d. random variables with the standard Gaussian distribution. In this case, Gamma approximations of Gaussian Wiener integrals corresponds to approximations in the second Wiener Chaos. We remark that Gamma approximations concern only Wiener chaoses of even orders since the third moment of a Wiener integral of odd degree is zero, while $\mathbb{E}\left[F(\nu)^{3}\right]=8 \nu>0$.

In [32] the authors provided the equivalent of Theorem 3.5 for $F(\nu)$-convergence, as summarized in the next statement.

Theorem 3.8. Let $d \geq 2$ be an even integer. If $X$ satisfies Assumption (1) and $\chi_{4}(X) \geq 0$, for every sequence of admissible kernels $f_{n}:[n]^{d} \rightarrow \mathbb{R}$ such that

$$
\lim _{n \rightarrow \infty} \mathbb{E}\left[Q_{\mathbf{X}}\left(f_{n}\right)^{2}\right]=\nu>0,
$$

the following statements are equivalent as $n \rightarrow \infty$ :

(i) $\mathbb{E}\left[Q_{\mathbf{X}}\left(f_{n}\right)^{4}\right]-12 \mathbb{E}\left[Q_{\mathbf{X}}\left(f_{n}\right)^{3}\right] \longrightarrow \mathbb{E}\left[F(\nu)^{4}\right]-12 \mathbb{E}\left[F(\nu)^{3}\right]=12 \nu^{2}-48 \nu ;$

(ii) $Q_{\mathbf{X}}\left(f_{n}\right) \stackrel{\text { Law }}{\longrightarrow} F(\nu)$.

Besides, the law of $X$ is universal for $F(\nu)$-approximations of homogeneous sums at the fixed order $d$, that is, $Q_{\mathbf{X}}\left(f_{n}\right) \stackrel{\text { Law }}{\longrightarrow} F(\nu)$ implies that $Q_{\mathbf{Z}}\left(f_{n}\right) \stackrel{\text { Law }}{\longrightarrow} F(\nu)$ for every sequence $\mathbf{Z}=\left\{Z_{i}\right\}_{i \geq 1}$ of i.i.d. centered random variables having unit variance.

Theorem 3.8 encompasses the findings in ([25], Thm. 1.2), where the first Fourth Moment-type statement for Gamma approximations of sequences of multiple Wiener integrals was provided, while the universality of Gaussian homogeneous sums w.r.t. $F(\nu)$-approximations was first established in ([28], Thm. 1.12), both for homogeneous sums with i.i.d. entries and with only independent entries.

Let us switch to the non-commutative probability setting, where the fourth-moment and universality phenomena have been studied as to free Poisson limits. Let $Z(\lambda)$ denote a centered free Poisson random variable of parameter $\lambda$, that is $\kappa_{1}(Z(\lambda))=0$ and $\kappa_{n}(Z(\lambda))=\lambda$ for all $n \geq 2$.

Stressing that free Poisson approximations can be established only in Wigner chaos of even order since, if $d$ is odd, $\varphi\left(Q_{\mathbf{S}}\left(f_{n}\right)^{3}\right)=0$ while $\varphi\left(Z(\lambda)^{3}\right)=\lambda>0$, Theorem 3.6 was adapted in [32] to deal also with free Poisson limits in the following terms.

Theorem 3.9. Let $d \geq 2$ be even. If $Y$ satisfies assumption (2) and $\kappa_{4}(Y) \geq 0$, for every sequence of admissible kernels $f_{n}:[n]^{d} \rightarrow \mathbb{R}$ such that

$$
\lim _{n \rightarrow \infty} \varphi\left(Q_{\mathbf{Y}}\left(f_{n}\right)^{2}\right)=\lambda,
$$

the following statements are equivalent in the limit:

(i) $Q_{\mathbf{Y}}\left(f_{n}\right) \stackrel{\text { Law }}{\longrightarrow} Z(\lambda)$;

(ii) $\varphi\left(Q_{\mathbf{Y}}\left(f_{n}\right)^{4}\right)-2 \varphi\left(Q_{\mathbf{Y}}\left(f_{n}\right)^{3}\right) \longrightarrow \varphi\left(Z(\lambda)^{4}\right)-2 \varphi\left(Z(\lambda)^{3}\right)=2 \lambda^{2}-\lambda$. 
Besides, the law of $Y$ is universal for free Poisson approximations of homogeneous sums at the order $d$, that is, $Q_{\mathbf{Y}}\left(f_{n}\right) \stackrel{\text { Law }}{\longrightarrow} Z(\lambda)$ implies that $Q_{\mathbf{W}}\left(f_{n}\right) \stackrel{\text { Law }}{\longrightarrow} Z(\lambda)$ for every other sequence $\mathbf{W}=\left\{W_{i}\right\}_{i \geq 1}$ of freely independent random variables satisfying assumption (2).

The first statement in Theorem 3.9 is a generalization for homogenous sums of the Fourth Moment Theorem for free Poisson limits of Wigner integrals first established in ([27], Thm. 1.4). Moreover, we remark that it contemplates also ([9], Thm. 1.5) when restricting to free Poisson homogeneous sums with fully symmetric coefficients.

As to universality, Theorem (3.9 extends [40], Thm. 3.15), from which one obtains, in particular, that semicircular and free Poisson homogeneous sums with fully symmetric coefficients behave universally also with respect to free Poisson convergence.

Remark 3.10. Another remarkable example of limit distribution that admits a fourth-moment type statement is the Tetilla distribution.

Let $\mathcal{T}$ denote a Tetilla distributed free random variable on a $W^{\star}$-probability space, that is $\mathcal{T}$ has the distribution of the standardized commutator between two freely independent semicircular elements $S_{1}, S_{2}$ :

$$
\mathcal{T} \stackrel{\text { Law }}{=} \frac{1}{\sqrt{2}}\left(S_{1} S_{2}+S_{2} S_{1}\right)
$$

In ([15], Thm. 1.1), the authors proved that for a sequence of Wigner integrals with (mirror) symmetric coefficients, Tetilla approximations can be assessed by controlling the corresponding convergence of the fourth and of the sixth moments. In particular, for semicircular homogeneous sums, it means that the moment conditions:

(i) $\varphi\left(Q_{S}\left(f_{n}\right)^{6}\right) \rightarrow \varphi\left(\mathcal{T}^{6}\right)$;

(ii) $\varphi\left(Q_{S}\left(f_{n}\right)^{4}\right) \rightarrow \varphi\left(\mathcal{T}^{4}\right)$,

are sufficient for the Tetilla approximation of the sequence $Q_{S}\left(f_{n}\right)$.

\subsection{Beyond: extensions and generalizations}

The present subsection is devoted to supply the reader with some bibliographic comments on topics related to the framework here investigated: in particular, with reference to other results on the fourth moment phenomenon and on universal limit theorems.

For instance, further results dealing with universality and Fourth Moment Theorems in the free setting include $[9,10,16,27]$, where the same phenomena were considered within the free Poisson algebra, and [40], where the results in [16] were extended to the multidimensional framework, entailing other instances of laws that are universal for semicircular and free Poisson approximation of homogeneous sums.

As to the commutative framework, among others we quote [38] for the analysis of the fourth moment and universality phenomenon on the discrete Poisson chaos (that will be given some special attention in the following). For elements of the Gaussian Wiener chaos, the reference [29,37] deals with normal approximation in the multidimensional setting, while [6] extends the fourth-moment condition for central convergence to higher moments conditions, that is, central convergence of multiple Wiener integrals can be assessed by the convergence of every pair of even moments. In addition, we would like to remark that the Fourth Moment Theorem has recently been approached also for Markov diffusion generators [5].

The analysis of the fourth moment phenomenon has been addressed also with respect to non-central convergence of Wiener integrals [25] as to Gamma limits, while the analysis of the corresponding universal behavior can be found in [28]. In this respect, Theorem 3.8 encompasses the framework of Gaussian homogeneous sums. For infinitely divisible laws [2], the fourth moment phenomenon has been explored in the classical, free, boolean and monotone probability settings. These results have been then supplemented with quantitative estimates for the Kolmogorov distance between infinitely divisible laws and the normal (resp. semicircular) laws, based on the distance between the corresponding fourth moments [3]. 
With reference to our third focus, recently it has been shown that such the equivalence between joint and component-wise convergence as to Gaussian approximations holds also for the Markov Chaos, by simply requiring an additional moment condition [11]. The reference also points out an updated account of other instances of Fourth Moment criteria beyond the framework of homogeneous sums. Other than in [37], the reader can found a quantitative analysis of the multidimensional Fourth Moment Theorem on Wiener Chaos in [29], while for an information-theoretical setting, the reader can consult [31], where entropic bounds are provided.

In the free probability setting, the analysis of the fourth moment phenomenon for non-linear functionals of a free Brownian motion started in [20], where the authors provided the non-commutative counterpart to the findings in [33] and deal with stochastic analysis via the free version of the Malliavin calculus, introduced in [8]. As already mentioned, the reference [27] has provided the non-central version of the Fourth Moment Theorem within the free Poisson algebra; further analysis on the free Poisson chaos have been run in ([10], Thm. 1.2) and [9], where the main result is that also free Poisson convergence of free Poisson integrals can be established with a fourth moment condition. Among several results, the author analyses the possibility of a transfer principle between Wigner and free Poisson chaoses, depending upon the parity of the involved orders.

Extensions of the Fourth Moment Theorem are provided in ([17], Thms. 1.7 and 3.1) for multiple integrals of symmetric kernels with respect to a $q$-Brownian motion, for non-negative values of $q$.

\section{The discrete Poisson Chaos}

Other than for Gaussian fields, another fertile ground for the fourth moment and the universality phenomena is the discrete Poisson chaos: the reference ([38], Thms. 3.2 and 3.4) focuses on normal approximations of Poisson homogeneous sums, while [36] considers Gamma limits.

Further works related to the Poisson chaos are [18,21], considering more general functionals of Poisson measures having the form of finite sums of multiple integrals with constant-sign kernels, with applications to geometric random graph, and [39] where the authors provided explicit bounds in CLTs for Poisson functionals, involving Stein's method and Malliavin calculus. Four moments conditions for Gamma convergence on the discrete Poisson chaos are analysed in [19].

As to the free Poisson chaos, the Fourth Moment Theorem for semicircular and free Poisson approximations have been provided in ([10], Thm. 1.2, and [9], Thm. 1.5) respectively. It is due to remark that ([9], Thm. 1.5) is a more complete statement since it applies also to free Poisson integrals of odd orders, while the strategy underlying Theorem 3.9 relies on the comparison with the corresponding semicircular homogeneous sums, and hence we need the assumption on the parity of $d$. For further universality results concerning free Poisson homogeneous sums, both with respect to central and free Poisson convergence, see [40].

However we remark that the Poisson homogeneous chaos should be considered special due to the lack of a transfer principle for central convergence between classical and free Poisson chaos, as highlighted in ([10], Thm. 1.2) with a counterexample. Simultaneously we remark that the Fourth Moment Theorem, as well as the universal behavior, within the classical Poisson Chaos, both with respect to central and Gamma convergence, are not encompassed by the findings of [32], which structurally depend on the assumption $\mathbb{E}\left[X^{3}\right]=0$.

\subsection{Some examples}

Theorems 3.5 and 3.8 supply us with several examples of laws that satisfy the Fourth Moment Theorem and are universal for central and Gamma convergence in the sense of Definition 2.3, encompassing all the known instances of Fourth Moment and universality phenomena (exception due to the Poisson chaos, as we shall discuss in a subsequent subsection). Here are few.

(1) Let $X_{1}, X_{2}$ be independent random variables satisfying assumption (1) and such that $\chi_{4}\left(X_{1}\right), \chi_{4}\left(X_{2}\right) \geq 0$. Then $Z=X_{1}+X_{2}$ satisfies in turn assumption (1) and $\chi_{4}(Z) \geq 0$ (due to the additivity property of cumulants), and hence satisfies the Fourth Moment Theorem. As to multiplicative convolution, $W:=X_{1} X_{2}$ satisfies assumption (1) as well. By virtue of the moment-cumulant formula, $\chi_{4}(W)=\mathbb{E}\left[X_{1}^{4}\right] \mathbb{E}\left[X_{2}^{4}\right]-3$ and 
hence, according to Theorem 3.5, for $W$ to satisfy the Fourth Moment Theorem it is sufficient that that at least one of the $X_{i}$ 's satisfies $\chi_{4}\left(X_{i}\right) \geq 0$.

(2) Every random variable $X$, centered and with unit variance, whose law is infinitely divisible with respect to additive convolution, satisfies $\chi_{4}(X)=\mathbb{E}\left[X^{4}\right]-3 \geq 0$ (see for instance ([3], Prop. A1). Hence, if $X$ is infinitely divisible and satisfies assumption (1), the Fourth Moment Theorem for homogeneous sums $Q_{\mathbf{X}}\left(f_{n}\right)$ holds at any order $d \geq 2$. The same necessary condition on the kurtosis of $X$ has been exploited to study the non-classical infinite divisibility of power semicircular distributions in [4].

(3) Let $N \sim \mathcal{N}(0,1)$ and, for $k \geq 1$, let $H_{k}(x)$ denote the $k$ th Hermite polynomial defined via the recursive relation:

$$
H_{0}=1, \quad H_{1}(x)=x, \quad H_{m+1}=x H_{m}(x)-m H_{m-1}(x) \forall m \geq 1 .
$$

Then, exploiting a standard notation for partitions of a set (see e.g. [35]),

$$
\mathbb{E}\left[H_{k}(N)^{4}\right]=\left|\mathcal{P}_{2}^{\star}\left(k^{\otimes 4}\right)\right| \geq 3
$$

where $\mathcal{P}_{2}^{\star}\left(k^{\otimes 4}\right)$ denotes the set of pairing partitions $\sigma$ of the set $[4 k]$ such that every block of $\sigma$ intersect each block of the partition:

$$
k^{\otimes 4}:=\{\{1, \ldots, k\},\{k+1, \ldots, 2 k\}, \ldots,\{2 k+1, \ldots, 3 k\},\{3 k+1, \ldots, 4 k\}\}
$$

in at most one element. Since $\mathbb{E}\left[H_{k}(N)^{3}\right]=0$ if $k$ is odd, for $X=H_{k}(N)$, Theorem 3.5 applies: the techniques so far established do not allow us to infer that the assumption on the third moment can be dropped.

As to the non-commutative setting, Theorems 3.6 and 3.9 establishe that the Fourth Moment Theorem, along with the universality phenomenon, applies for instance, in the following cases:

(1) Every random variable $Y$ satisfying assumption (2) and whose law is infinitely divisible with respect to the additive free convolution, satisfies $\kappa_{4}(Y)=\varphi\left(Y^{4}\right)-2 \geq 0$ (see again [3], Prop. A1). Hence, every freely infinitely divisible law satisfies the Fourth Moment Theorem (and the universality) as to semicircular and free Poisson approximations of homogeneous sums, at any order $d \geq 2$.

(2) For $k \geq 1$, if $U_{k}(x)$ denotes the $k$ th Chebyshev polynomial (of the second kind), defined via the recursive relation:

$$
U_{0}=1, \quad U_{1}(x)=x, \quad U_{m+1}(x)=x U_{m}(x)-U_{m-1}(x) \quad \forall m \geq 1 .
$$

If $S \sim \mathcal{S}(0,1)$, then:

$$
\varphi\left(U_{k}(S)^{4}\right)=\left|\mathcal{N C}_{2}^{\star}\left(k^{\otimes 4}\right)\right| \geq 2,
$$

where $\mathcal{N C}_{2}^{\star}\left(k^{\otimes 4}\right)$ denotes the set of the non-crossing pairings $\sigma$ of the set [4k], such that in each block of $\sigma$, there is at most one element of every block of the interval partition $k^{\otimes 4}$ defined in (5). Note that the universality of the law of $U_{k}(S)$ for semicircular and free Poisson approximations (for even degrees) of homogeneous sums can be also established via the approach developed in [40].

(3) Let $\mathcal{T}$ be a Tetilla-distributed random variable, as in (4). Since $\kappa_{4}(\mathcal{T})=\frac{1}{2}>0, \mathcal{T}$ satisfies both the Fourth Moment Theorem and the universality property for semicircular and free Poisson approximations of homogeneous sums, at any order $d \geq 2$ (see [15]).

(4) Let $X \sim \mathcal{G}_{q}(0,1)$, with $\mathcal{G}_{q}(0,1)$ denoting the $q$-Gaussian distribution - that we assume to be defined on an adequate non-commutative probability space $\left(\mathscr{A}_{q}, \varphi_{q}\right)$. Then, $\kappa_{4}(X)=\varphi_{q}\left(X^{4}\right)-2=q$, and hence, if $q \in[0,1], X$ satisfies the Fourth Moment Theorem and the law $\mathcal{G}_{q}(0,1)$ is universal at any order $d \geq 2$ for semicircular and free Poisson approximations of free homogeneous sums (for $d$ even). Alternatively, the fourth moment and the universality phenomena for $X$ can be deduced from the fact that, for positive values of $q$, the $q$-Gaussian distribution is also freely infinitely divisible [1]. 


\subsection{Multidimensional central limit theorem}

Gaussian and Semicircular homogeneous sums carry nice properties also in the multidimensional setting, due to the property that component-wise and joint convergence for the CLT are indeed equivalent, as we will recall with some details in the present subsection. We present here a concise list of the most relevant results matching with our purposes, siding every results in the commutative framework with its free version, always in order to enhance similarities as well as differences.

We start with recalling ([28], Thm. 7.1]), where the authors have provided an explicit error bound for the distance in law between a random vector of the type $\left(Q_{\boldsymbol{X}}\left(f_{n}^{(1)}\right), \ldots, Q_{\boldsymbol{X}}\left(f_{n}^{(m)}\right)\right)$ and its Gaussian counterpart $\left(Q_{N}\left(f_{n}^{(1)}\right), \ldots, Q_{N}\left(f_{n}^{(m)}\right)\right)$. For the sake of completeness, it is worth to underline that a first attempt of extending Theorem 3.2 to the multidimensional case has been carried out in ([22], Thm. 4.1), in the case one of the sequences lives on a discrete probability space.

Theorem 3.11. Let $m, d \geq 1$. Let $\boldsymbol{X}=\left\{X_{i}\right\}_{i \geq 1}$ be a sequence of centered independent random variables, with unit variance, whose third moments are uniformly bounded (namely, such that there exists $\beta>0$ such that $\left.\sup \mathbb{E}\left[\left|X_{i}\right|^{3}\right]<\beta\right)$. For $j=1, \ldots, m$, let $f_{n}^{(j)}:[n]^{d} \rightarrow \mathbb{R}$ be an admissible kernel according to Definition 2.1. If $i \geq 1$

$\boldsymbol{N}=\left\{N_{i}\right\}_{i \geq 1}$ denotes a sequence of i.i.d. standard Gaussian random variables, for every thrice differentiable function $\psi: \mathbb{R}^{m} \rightarrow \mathbb{R}$, with $\left\|\psi^{\prime \prime \prime}\right\|_{\infty}<\infty$, there exists a constant $C=C(\beta, m, d, \psi)$ such that:

$$
\left|\mathbb{E}\left[\psi\left(\boldsymbol{Q}_{n}(\boldsymbol{X})\right)\right]-\mathbb{E}\left[\psi\left(\boldsymbol{Q}_{n}(\boldsymbol{N})\right)\right]\right| \leq C \sqrt{\max _{j=1, \ldots, m} \tau_{n}\left(f_{n}^{(j)}\right)},
$$

where $\boldsymbol{Q}_{n}(\boldsymbol{X})=\left(Q_{\boldsymbol{X}}\left(f_{n}^{(1)}\right), \ldots, Q_{\boldsymbol{X}}\left(f_{n}^{(m)}\right)\right)$, and $\boldsymbol{Q}_{n}(\boldsymbol{N})$ denotes its Gaussian counterpart.

The findings established with ([40], Thm. 3.2) provide a general multidimensional version of Theorem 3.2 in the free probability setting. Here the invariance principle will be formulated via Theorem 3.12 for estimating the proximity in law between vectors of free homogeneous sums. It is worth to remark that Theorem 3.12 has been originally formulated for more general objects than homogeneous sums, therein called Chebyshev sums, and that in the above simplified formulation for homogeneous sums, it encompasses ([16], Thm. 1.3) corresponding to $p=1$.

Theorem 3.12. Let $\boldsymbol{X}=\left\{X_{i}\right\}_{i \geq 1}$ and $\boldsymbol{Y}=\left\{Y_{j}\right\}_{j \geq 1}$ be sequences of freely independent random variables, centered and with unit variance, freely independent between each other. Assume further that $\boldsymbol{X}$ and $\boldsymbol{Y}$ are composed of random variables with uniformly bounded moments, that is, for every integer $r \geq 1$,

$$
\sup _{i \geq 1} \varphi\left(\left|X_{i}\right|^{r}\right)<\infty \quad\left(\text { resp. } \sup _{i \geq 1} \varphi\left(\left|Y_{i}\right|^{r}\right)<\infty\right) .
$$

For every integer $k \geq 1$, for every choice of $\boldsymbol{m}_{s}=\left(m_{s, 1}, \ldots, m_{s, p}\right) \in \mathbb{N}^{p}$ for $s=1, \ldots, k$, if $\boldsymbol{Q}_{n}(\boldsymbol{Y})=$ $\left(Q_{\boldsymbol{Y}}\left(f_{n}^{(1)}\right), \ldots, Q_{\boldsymbol{Y}}\left(f_{n}^{(p)}\right)\right)$ denotes a vector of homogeneous sums with admissible kernel $f_{n}^{(j)}:[n]^{d} \rightarrow \mathbb{R}$ for every $j=1, \ldots, p$, then:

$$
\begin{aligned}
\varphi\left(\boldsymbol{Q}_{n}(\boldsymbol{X})^{\boldsymbol{m}_{1}} \boldsymbol{Q}_{n}(\boldsymbol{X})^{\boldsymbol{m}_{2}} \cdots \boldsymbol{Q}_{n}(\boldsymbol{X})^{\boldsymbol{m}_{k}}\right) & -\varphi\left(\boldsymbol{Q}_{n}(\boldsymbol{Y})^{\boldsymbol{m}_{1}} \boldsymbol{Q}_{n}(\boldsymbol{Y})^{\boldsymbol{m}_{2}} \cdots \boldsymbol{Q}_{n}(\boldsymbol{Y})^{\boldsymbol{m}_{k}}\right) \\
& =\mathcal{O}\left(\max _{j=1, \ldots, p}\left(\tau_{n}^{(j)}\right)^{\frac{1}{2}}\right),
\end{aligned}
$$

where $\tau_{n}^{(j)}=\max _{i=1, \ldots, n} \operatorname{Inf}_{i}\left(f_{n}^{(j)}\right)$ and where for $\boldsymbol{m}=\left(m_{1}, \ldots, m_{p}\right) \in \mathbb{N}^{p}$ we have used the standard multi-index notation

$$
\boldsymbol{Q}_{n}(\boldsymbol{Y})^{\boldsymbol{m}}:=Q_{\boldsymbol{Y}}\left(f_{n}^{(1)}\right)^{m_{1}} Q_{\boldsymbol{Y}}\left(f_{n}^{(2)}\right)^{m_{2}} \cdots Q_{\boldsymbol{Y}}\left(f_{n}^{(p)}\right)^{m_{p}}
$$


We have recalled all the tools needed to approach our third task, that is, the equivalence between joint and component-wise central convergence for vectors of homogeneous sums.

Let us start with the commutative setting: the subsequent Theorem 3.13 recalls ([37], Prop. 2), where it is shown that for vectors of the type $\left(Q_{N}\left(f_{n}^{(1)}\right), \ldots, Q_{N}\left(f_{n}^{(m)}\right)\right)$, joint convergence towards the multidimensional normal distribution is equivalent to component-wise central convergence. Note that the original statement does not concern exclusively vectors of Gaussian homogeneous sums, but deals with vectors of multiple Wiener integrals of symmetric functions in full generality.

In the following, let $V=\left(V_{i, j}\right)_{i, j=1, \ldots, m}$ denote a real valued, positive definite, symmetric matrix, and let $\mathcal{N}(0, V)$ denote the $m$-dimensional Gaussian distribution with covariance matrix given by $V$. Similarly, assume that $\left(s_{1}, \ldots, s_{m}\right)$ denotes a semicircular system with covariance determined by $V$. As above, $\boldsymbol{S}=\left\{S_{i}\right\}_{i \geq 1}$ will denote a sequence of freely independent standard semicircular random variables, and $\boldsymbol{N}=\left\{N_{i}\right\}_{i \geq 1}$ a sequence of independent standard Gaussian random variables, taken on suitable free and classical probability spaces, respectively.

Theorem 3.13. Let $d \geq 2$ and $m \geq 1$. For every $j=1, \ldots, m$, let $Q_{\boldsymbol{X}}\left(f_{n}^{(j)}\right)$ be a sequence of homogeneous sums of degree $d$, with $f_{n}^{(j)}:[n]^{d} \rightarrow \mathbb{R}$ symmetric kernel, vanishing on diagonals, such that:

$$
\lim _{n \rightarrow \infty} \mathbb{E}\left[Q_{\boldsymbol{X}}\left(f_{n}^{(j)}\right) Q_{\boldsymbol{X}}\left(f_{n}^{(i)}\right)\right]=V_{i, j} \quad \forall i, j=1, \ldots, m .
$$

Then, the following statements are equivalent as $n \rightarrow \infty$ :

(i) $Q_{N}\left(f_{n}^{(j)}\right) \stackrel{\text { Law }}{\longrightarrow} \mathcal{N}\left(0, V_{j, j}\right)$ for every $j=1, \ldots, m$;

(ii) $\left(Q_{\boldsymbol{N}}\left(f_{n}^{(1)}\right), \ldots, Q_{\boldsymbol{N}}\left(f_{n}^{(m)}\right)\right) \stackrel{\text { Law }}{\longrightarrow} \mathcal{N}(0, V)$.

Theorem 3.13 has found a free counterpart with ([30], Thm. 1.3): even if the original statement deals with vectors of Wigner stochastic integrals in full generality, the statement here is recalled only for semicircular homogeneous sums.

Theorem 3.14. For $d \geq 2$ and $m \geq 1$, let $f_{n}^{(j)}:[n]^{d} \rightarrow \mathbb{R}$ be a mirror symmetric function for every $j=$ $1, \ldots, m$. Let the above notation prevail, and assume that for $i, j=1, \ldots, m$,

$$
\lim _{n \rightarrow \infty} \varphi\left(Q_{\mathbf{S}}\left(f_{n}^{(i)}\right) Q_{\mathbf{S}}\left(f_{n}^{(j)}\right)\right)=V_{i, j} \quad \forall i, j=1, \ldots, m . .
$$

Then, the following statements are equivalent as $n \rightarrow \infty$ :

(i) $Q_{\mathbf{S}}\left(f_{n}^{(j)}\right) \stackrel{\text { Law }}{\longrightarrow} s_{j}$;

(ii) $\left(Q_{\mathbf{S}}\left(f_{n}^{(1)}\right), \ldots, Q_{\mathbf{S}}\left(f_{n}^{(m)}\right)\right) \stackrel{\text { Law }}{\longrightarrow}\left(s_{1}, \ldots, s_{m}\right)$.

The striking parallelism between the Gaussian and the Semicircular homogeneous chaoses can be formally explained via the Wiener-Wigner transfer principle (see [30], Thm. 1.6), that is recalled in the following statement only for homogeneous sums (always in line with our focus).

Theorem 3.15. Let $d \geq 1$ and $m \geq 1$ be fixed integers. For every $j=1, \ldots, m$, let $f_{n}^{(j)}:[n]^{d} \rightarrow \mathbb{R}$ be an admissible kernel, and assume that, for every $i, j=1, \ldots, m$ :

$$
\lim _{n \rightarrow \infty} d ! \varphi\left(Q_{S}\left(f_{n}^{(i)}\right) Q_{S}\left(f_{n}^{(j)}\right)\right)=\lim _{n \rightarrow \infty} \mathbb{E}\left[Q_{N}\left(f_{n}^{(i)}\right) Q_{N}\left(f_{n}^{(j)}\right)\right]=V_{i, j} .
$$

Then the following statements are equivalent as $n \rightarrow \infty$ :

(i) $\left(\sqrt{d !} Q_{S}\left(f_{n}^{(1)}\right), \ldots, \sqrt{d !} Q_{S}\left(f_{n}^{(m)}\right)\right) \stackrel{\text { Law }}{\longrightarrow}\left(s_{1}, \ldots, s_{m}\right)$;

(ii) $\left(Q_{N}\left(f_{n}^{(1)}\right), \ldots, Q_{N}\left(f_{n}^{(m)}\right)\right) \stackrel{\text { Law }}{\longrightarrow} \mathcal{N}(0, V)$. 


\section{Applications: some neW Results}

We have already underlined in the Introduction that the ultimate goal of our overview is to revisit the reference [32] within a multidimensional perspective.

As matching pieces of a puzzle, we will now combine the results so far summarized in order to establish the multidimensional counterparts to Theorems 3.5 and 3.6 stated in terms of equivalence between component-wise and joint convergence. The goal is perfectioning the findings of [32] in a multidimensional setting, essaying to include also Theorems 3.13 and 3.14 in the unified framework provided with Theorems 3.5 and 3.6.

In particular, it will be shown how it is possible to exploit the findings of [32] in order to generalize the transfer principle stated with Theorem 3.15, with no computational nor theoretical efforts.

We start with the multidimensional version of Theorem 3.5, stated via Theorem 4.1: combining Theorems 3.5, 3.11 and 3.13 , it is possible to conclude that the equivalence between joint and component-wise convergence for normal approximations of random vectors of the form $\left(Q_{\boldsymbol{X}}\left(f_{n}^{(1)}\right), \ldots, Q_{\boldsymbol{X}}\left(f_{n}^{(m)}\right)\right)$ always holds true under the assumptions $\mathbb{E}\left[X^{3}\right]=0$ and $\mathbb{E}\left[X^{4}\right] \geq 3$.

Theorem 4.1. Fix $m \geq 1$ and $d \geq 2$. Let $\boldsymbol{X}=\left\{X_{i}\right\}_{i \geq 1}$ be a sequence of independent copies of a random variable $X$ verifying assumption (1) and such that $\chi_{4}(X)=\mathbb{E}\left[X^{4}\right]-3 \geq 0$. For every $j=1, \ldots, m$, let $Q_{\boldsymbol{X}}\left(f_{n}^{(j)}\right)$ be a sequence of homogeneous sums of degree $d$, with $f_{n}^{(j)}:[n]^{d} \rightarrow \mathbb{R}$ admissible kernel, such that:

$$
\lim _{n \rightarrow \infty} \mathbb{E}\left[Q_{\boldsymbol{X}}\left(f_{n}^{(i)}\right) Q_{\boldsymbol{X}}\left(f_{n}^{(j)}\right)\right]=V_{i, j} \quad \forall i, j=1, \ldots, m .
$$

The following statements are equivalent as $n \rightarrow \infty$ :

(i) $Q_{\boldsymbol{X}}\left(f_{n}^{(j)}\right) \stackrel{\text { Law }}{\longrightarrow} \mathcal{N}\left(0, V_{j, j}\right)$ for every $j=1, \ldots, m$;

(ii) $\left(Q_{\boldsymbol{X}}\left(f_{n}^{(1)}\right), \ldots, Q_{\boldsymbol{X}}\left(f_{n}^{(m)}\right)\right) \stackrel{\text { Law }}{\longrightarrow} \mathcal{N}(0, V)$.

Proof. It is sufficient to prove that $(i) \Rightarrow(i i)$, since the reverse implication is obvious under our assumptions.

Assume that (i) occurs. Under the assumption $E\left[X^{4}\right] \geq 3$ and by virtue of Theorem $3.5, X$ satisfies the Fourth Moment Theorem and its law is universal at the order $d$ for normal approximations of homogeneous sums of degree $d$, implying, in particular, that:

$$
Q_{N}\left(f_{n}^{(j)}\right) \stackrel{\text { Law }}{\longrightarrow} \mathcal{N}\left(0, V_{j, j}\right) \text { for every } j=1, \ldots, m,
$$

for a sequence $\boldsymbol{N}$ of independent standard Gaussian random variables. Besides, for every $j=1, \ldots, m, \tau_{n}^{(j)}=$ $\max _{i=1} \operatorname{Inf}_{i}\left(f_{n}^{(j)}\right) \longrightarrow 0$ in the limit $n \rightarrow \infty$. Then, remarking that:

$$
\mathbb{E}\left[Q_{\boldsymbol{X}}\left(f_{n}^{(j)}\right) Q_{\boldsymbol{X}}\left(f_{n}^{(i)}\right)\right]=\mathbb{E}\left[Q_{\boldsymbol{N}}\left(f_{n}^{(j)}\right) Q_{\boldsymbol{N}}\left(f_{n}^{(i)}\right)\right] \quad \forall i, j=1, \ldots, m,
$$

by virtue of Theorem 3.11 it follows that $\left(Q_{\boldsymbol{N}}\left(f_{n}^{(1)}\right), \ldots, Q_{\boldsymbol{N}}\left(f_{n}^{(m)}\right)\right)$ and $\left(Q_{\boldsymbol{X}}\left(f_{n}^{(1)}\right), \ldots, Q_{\boldsymbol{X}}\left(f_{n}^{(m)}\right)\right)$ are asymptotically close in distribution. Finally, the conclusion follows by applying Theorem 3.13.

With a similar technique, the combination between Theorems 3.12, 3.6 and 3.14 allows us to prove straightforwardly that Theorem 3.14 itself can be extended to all random variables with non-negative free kurtosis, providing therefore the free analogue to Theorem 4.1.

Theorem 4.2. Fix $m \geq 1$ and $d \geq 2$. Let $\boldsymbol{Y}=\left\{Y_{i}\right\}_{i \geq 1}$ be a sequence of freely independent copies of a random variable $Y$ verifying assumption (1) and such that $\kappa_{4}(Y)=\varphi\left(Y^{4}\right)-2 \geq 0$. For every $j=1, \ldots, m$, let $Q_{\mathbf{Y}}\left(f_{n}^{(j)}\right)$ be a sequence of homogeneous sums of degree $d$, with symmetric and vanishing on diagonals kernels $f_{n}^{(j)}:[n]^{d} \rightarrow \mathbb{R}$ such that:

$$
\lim _{n \rightarrow \infty} \varphi\left(Q_{\boldsymbol{Y}}\left(f_{n}^{(j)}\right) Q_{\boldsymbol{Y}}\left(f_{n}^{(i)}\right)\right)=V_{i, j} \quad \forall i, j=1, \ldots, m
$$


The following statements are equivalent as $n \rightarrow \infty$ :

(i) $Q_{\boldsymbol{Y}}\left(f_{n}^{(j)}\right) \stackrel{\text { Law }}{\longrightarrow} s_{j}$ for every $j=1, \ldots, m$;

(ii) $\left(Q_{\boldsymbol{Y}}\left(f_{n}^{(1)}\right), \ldots, Q_{\boldsymbol{Y}}\left(f_{n}^{(m)}\right)\right) \stackrel{\text { Law }}{\longrightarrow}\left(s_{1}, \ldots, s_{m}\right)$.

Proof. It is sufficient to prove that $(i) \Rightarrow(i i)$, since the reverse implication is obvious.

Assume that (i) occurs. Under the assumption $\varphi\left(Y^{4}\right) \geq 2$ and by virtue of Theorem 3.6, $Y$ satisfies the Fourth Moment Theorem and its law is universal for semicircular approximations of homogeneous sums, at the given order $d$. In particular one has $Q_{\mathbf{S}}\left(f_{n}^{(j)}\right) \stackrel{\text { Law }}{\longrightarrow} s_{j}$, and $\tau_{n}^{(j)}=\max _{i=1, \ldots, n} \operatorname{Inf}_{i}\left(f_{n}^{(j)}\right) \longrightarrow 0$ for every $j=1, \ldots, m$. Finally, since

$$
\varphi\left(Q_{\boldsymbol{Y}}\left(f_{n}^{(j)}\right) Q_{\boldsymbol{Y}}\left(f_{n}^{(i)}\right)\right)=\varphi\left(Q_{\boldsymbol{S}}\left(f_{n}^{(j)}\right) Q_{\boldsymbol{S}}\left(f_{n}^{(i)}\right)\right) \quad \forall i, j=1, \ldots, m
$$

by virtue of Theorem 3.12 it follows that the vectors $\left(Q_{\boldsymbol{S}}\left(f_{n}^{(1)}\right), \ldots, Q_{\boldsymbol{S}}\left(f_{n}^{(m)}\right)\right)$ and $\left(Q_{\boldsymbol{Y}}\left(f_{n}^{(1)}\right), \ldots, Q_{\boldsymbol{Y}}\left(f_{n}^{(m)}\right)\right)$ are asymptotically close in distribution: hence the conclusion follows by Theorem 3.14.

In conclusion, in light of Theorems 4.1 and 4.2, Theorem 3.15 can be completely generalized to a transfer principle for central convergence between homogeneous sums $Q_{\mathbf{X}}\left(f_{n}\right)$, with $X$ satisfying assumption (1) and with non-negative kurtosis, over a classical probability space, and free homogeneous sums $\sqrt{d !} Q_{\mathbf{Y}}\left(f_{n}\right)$, with $Y$ satisfying assumption (2) and with non-negative free kurtosis, over a free probability space $(\mathcal{A}, \varphi)$. Note that, since we are analysing the occurrence of the fourth moment phenomenon along with the universality property, we need to set $d \geq 2$.

Theorem 4.3. Set $d \geq 2$. Let $X$ be a random variable satisfying assumption (1) and such that $\mathbb{E}\left[X^{4}\right] \geq 3$, and $Y$ be a free random variable satisfying assumption $(2)$ and $\varphi\left(Y^{4}\right) \geq 2$. For $m \geq 1$, let $f_{n}^{(j)}:[n]^{d} \rightarrow \mathbb{R}$ be a symmetric, vanishing on diagonal kernel for every $j=1, \ldots, m$, such that for all $i, j=1, \ldots, m$ :

$$
\lim _{n \rightarrow \infty} d ! \varphi\left(Q_{\mathbf{Y}}\left(f_{n}^{(i)}\right) Q_{\mathbf{Y}}\left(f_{n}^{(j)}\right)\right)=\frac{1}{d !} \lim _{n \rightarrow \infty} \mathbb{E}\left[Q_{\mathbf{X}}\left(f_{n}^{(i)}\right) Q_{\mathbf{X}}\left(f_{n}^{(j)}\right)\right]=V_{i, j}
$$

Then the following conditions are equivalent as $n \rightarrow \infty$ :

(i) $\left(Q_{\mathbf{X}}\left(f_{n}^{(1)}\right), \ldots, Q_{\mathbf{X}}\left(f_{n}^{(m)}\right)\right) \stackrel{\operatorname{Law}}{\longrightarrow} \mathcal{N}(0, V)$;

(ii) $\left(\sqrt{d !} Q_{\mathbf{Y}}\left(f_{n}^{(1)}\right), \ldots, \sqrt{d !} Q_{\mathbf{Y}}\left(f_{n}^{(m)}\right)\right) \stackrel{\operatorname{Law}}{\longrightarrow}\left(s_{1}, \ldots, s_{m}\right)$.

Proof. Assume first that $(i)$ holds: in particular, $Q \mathbf{X}\left(f_{n}^{(j)}\right) \stackrel{\text { Law }}{\longrightarrow} \mathcal{N}\left(0, V_{j, j}\right)$ for all $j=1, \ldots, m$, implying in turn that $Q_{\mathbf{N}}\left(f_{n}^{(j)}\right) \stackrel{\text { Law }}{\longrightarrow} \mathcal{N}\left(0, V_{j, j}\right)$ by virtue of Theorem 3.5. Then, from Theorem 3.13 it follows that:

$$
\left(Q_{\mathbf{N}}\left(f_{n}^{(1)}\right), \ldots, Q_{\mathbf{N}}\left(f_{n}^{(m)}\right)\right) \stackrel{\text { Law }}{\longrightarrow} \mathcal{N}(0, V)
$$

which is, by virtue of ([30], Thm. 1.6), equivalent to the joint convergence:

$$
\left(\sqrt{d !} Q_{\mathbf{S}}\left(f_{n}^{(1)}\right), \ldots, \sqrt{d !} Q_{\mathbf{S}}\left(f_{n}^{(m)}\right)\right) \stackrel{\text { Law }}{\longrightarrow}\left(s_{1}, \ldots, s_{m}\right) .
$$

Finally, Theorem 3.14 implies that $\sqrt{d !} Q_{\mathbf{Y}}\left(f_{n}^{(j)}\right) \stackrel{\text { Law }}{\longrightarrow} s_{j}$ for all $j=1, \ldots, m$ and the conclusion follows by Theorem 4.2.

To prove the reverse implication, start with Theorem 3.6 and consider Theorem 4.1 instead of Theorems 3.5 and 4.2 , respectively. 


\section{CONCluding REMARKS}

The original motivation for the present work was to summarize all the necessary background needed to face the multidimensional extension to [32], by providing some instances of results that can be effectively and simply derived with the tools so far available.

We stress that to the best of our knowledge there is no result indicating the equivalence between componentwise and joint convergence for Poisson limits, and hence our strategy cannot deal with Poisson approximations to state the correspondent version of Theorem 4.3.

As a final outcome of our overview, we wish to advance a possible motivation for the special case of the Poisson Chaos, that is lacking of a transfer principle. Due to the assumption $\mathbb{E}\left[X^{3}\right]=0$ in the statement of Theorem 3.5, the setting worked out in the present note as well as in [32] does not fit the Poisson homogeneous chaos. In view of the transfer principle provided with Theorem 4.3, this failure is consistent with the lack of a transfer principle, for central convergence, between classical and free Poisson chaos, as highlighted with a counterexample in [10].

\section{REFERENCES}

[1] M. Anshelevich, S.T. Belinschi, M. Bozejko and F. Lehner, Free infinite divisibility for $q$-Gaussians. Math. Res. Lett. 17 (2010) 905-916.

[2] O. Arizmendi, Convergence of the fourth moment and infinite divisibility. Probab. Math. Stat. 33 (2013) $201-212$.

[3] O. Arizmendi and A. Jaramillo, Convergence of the fourth moment and infinite divisibility: quantitative estimates. Electron. Commun. Probab. 19 (2014) 1-12.

[4] O. Arizmendi and V. Pérez-Abreu, On the non-classical infinite divisibility of power semicircle distributions. Commun. Stochastic Anal. 4 (2010) 161-178.

[5] E. Azmoodeh, S. Campese and G. Poly, Fourth Moment Theorems for Markov Diffusion Generators. J. Funct. Anal. 266 (2014) 2341-2359.

[6] E. Azmoodeh, D. Malicet, G. Mijoule and G. Poly, Generalization of the Nualart-Peccati criterion. Ann. Probab. 44 (2016) 924-954.

[7] B. Bhattacharya, P. Diaconis and S. Mukherjee, Universal limit theorems in graph coloring problems with connections to extremal combinatorics. Preprint arXiv: 1310.2336 (2014).

[8] P. Biane and R. Speicher, Stochastic analysis with respect to free Brownian motion and analysis on Wigner space. Probab. Theory Relat. Fields 112 (1998) 373-409.

[9] S. Bourguin, Poisson convergence on the free Poisson algebra. Bernoulli 21 (2015) 2139-2156.

[10] S. Bourguin and G. Peccati, Semicircular limits on the free Poisson chaos: counterexamples to a transfer principle. J. Funct. Anal. 267 (2013) 963-997.

[11] S. Campese, I. Nourdin, G. Peccati and G. Poly, Multivariate Gaussian approximation on Markov chaoses. Preprint arXiv:1510.02105 (2015).

[12] L.H.Y. Chen, Stein meets Malliavin in Normal Approximation. Acta Math. Vietnamica 40 (2015) 205.

[13] L.H.Y. Chen and G. Poly, Stein's method, Malliavin calculus, Dirichlet forms and the fourth moment theorem. Festschrift Masatoshi Fukushima. Edited by Z.-Q. Chen, N. Jacob, M. Takeda and T. Uemura. In vol. 17 of Interdiscipl. Math. Sci. (2015) 107-130.

[14] P. de Jong, A central limit theorem for generalized multilinear forms. J. Multivar. Anal. 34 (1987) 275-289.

[15] A. Deya and I. Nourdin, Convergence of Wigner integrals to the Tetilla law. ALEA, Lat. Am. J. Probab. Math. Stat. 9 (2012) 101-127.

[16] A. Deya and I. Nourdin, Invariance principles for homogeneous sums of free random variables. Bernoulli 20 (2013) 586-603.

[17] A. Deya, S. Norredine and I. Nourdin, Fourth Moment Theorem and q-Brownian Motion. Commun. Math. Phys. 321 (2013) 113-134.

[18] P. Eichelsbacher and C. Thäle, New Berry-Esseen bounds for non-linear functionals of Poisson random measures. Electron. J. Probab. 19 (2014) 1-25.

[19] T. Fissler and C. Thäle, A four moment theorem for Gamma limits on a Poisson chaos. Preprint arXiv:1502.01568 (2015).

[20] T. Kemp, I. Nourdin, G. Peccati and R. Speicher, Wigner Chaos and the fourth moment. Ann. Probab. 40 (2011) $1577-1635$.

[21] R. Lachiéze-Rey and G. Peccati, Fine Gaussian fluctuations on the Poisson space, I: contractions, cumulants and geometric random graphs. Electron. J. Probab. 18 (2013) 1-32.

[22] E. Mossel, Gaussian bounds for noise correlation of functions. Geometric Funct. Anal. 19 (2010) $1713-1756$.

[23] E. Mossel, R. O'Donnell and K. Oleszkiewicz, Noise stability of functions with low influences: invariance and optimality. Ann. Math. 171 (2010) 295-341.

[24] A. Nica and R. Speicher, Lectures on the Combinatorics of Free Probability. Cambridge University Press (1990).

[25] I. Nourdin and G. Peccati, Noncentral convergence of multiple integrals. Ann. Probab. 37 (2009) 1412-1426. 
[26] I. Nourdin and G. Peccati, Normal approximations with Malliavin calculus: from Stein's method to universality. Cambridge Tracts in Mathematics. Cambridge University Press (2012).

[27] I. Nourdin and G. Peccati. Poisson approximations on the free Wigner chaos. Ann. Probab. 41 (2013) $2709-2723$.

[28] I. Nourdin, G. Peccati and G. Reinert, Invariance principles for homogeneous sums: universality of Gaussian Wiener Chaos. Ann. Probab. 38 (2010) 1947-1985.

[29] I. Nourdin, G. Peccati and A. Reveillac, Multivariate normal approximation using Stein's method and Malliavin calculus. Ann. Inst. Henri Poincaré, Probab. Stat. 46 (2010) 45-58.

[30] I. Nourdin, G. Peccati and R. Speicher, Multidimensional semicircular limits on the free Wigner Chaos. Ascona Proceedings 2011. In vol. 67 of Progress in Probability (2013) 211-221.

[31] I. Nourdin, G. Peccati and Y. Swan, Entropy and the fourth moment phenomenon. J. Funct. Anal. 266 (2014) $3170-3207$.

[32] I. Nourdin, G. Peccati, G. Poly and R. Simone, Classical and free fourth moment theorems: universality and thresholds. J. Theoret. Probab. 29 (2016) 653-680.

[33] D. Nualart and G. Peccati, Central limit theorems for sequences of multiple stochastic integrals. Ann. Probab. 33 (2005) 177-193.

[34] G. Peccati, Quantitative CLTs on a Gaussian space: a survey of recent developments. ESAIM: Proc. Suv. 44 (2014) 61-78.

[35] G. Peccati and M.S. Taqqu, Wiener Chaos: Moments, Cumulants and Diagrams. Springer-Verlag (2010).

[36] G. Peccati and Ch. Thaele, Gamma limits and U-statistics on the Poisson space. ALEA, Lat. Am. J. Probab. Math. Stat. 10 (2013) 525-560.

[37] G. Peccati and C. Tudor, Gaussian limits for vector-valued multiple stochastic integrals. In vol. XXXVIII, Séminaire de Probabilités (2005) 247-262.

[38] G. Peccati and C. Zheng, Universal Gaussian fluctuations on the discrete Poisson chaos. Bernoulli 20 (2013) $697-715$.

[39] G. Peccati, J.L. Solé, M.S. Taqqu and F. Utzet, Stein's method and normal approximation of Poisson functionals. Ann. Probab. 38 (2010) 443-478.

[40] R. Simone, Universality for free homogeneous sums in every dimension. ALEA, Lat. Am. J. Probab. Math. Stat. 12 (2015) $213-244$.

[41] D. Voiculescu, Symmetries of some reduced free product $C^{*}$-algebras. Operator algebras and their connection with topology and ergodic theory. Vol. 1132 of Lect. Notes Math. Springer (1985) 556-588. 